\title{
ELETROMANOMETRIA DO ESFÍNCTER SUPERIOR DO ESÔFAGO ANTES E APÓS PERFUSÃO ESOFÁGICA COM ÁCIDO CLORÍDRICO 0,1N. Estudo experimental no cão
}

\author{
José Vicente TAGLIARINI*, Maria Aparecida Coelho de Arruda HENRY** e \\ Onivaldo BRETAN***
}

RESUMO - Racional - A resposta do esfíncter esofágico superior ao refluxo gastroesofágico é controvertida. A perfusão esofágica com ácido clorídrico é modelo de estudo da ação do ácido o componente mais agressivo do refluxo sobre o esfincter. Objetivos - Estudar o efeito da acidificação esofágica sobre o esfíncter esofágico superior através da eletromanometria esofágica. - Material e Métodos - Em 30 cães adultos de ambos os sexos foram registrados estudos eletromanométricos do esôfago. A técnica utilizada foi a de puxada intermitente da sonda e perfusão contínua dos cateteres com água destilada. Estes exames permitiram as medidas da amplitude da pressão ( $\mathrm{mm} \mathrm{Hg}$ ) e do comprimento (cm) do esfincter superior do esôfago em condições basais (momento 1). Após esta fase, os animais foram submetidos a perfusão esofágica e divididos em 3 grupos de 10, na dependência da solução utilizada na perfusão e do momento do estudo: Grupo 1: perfusão esofágica com água destilada e estudos eletromanométricos realizados aos 15 minutos (momento 2) e 30 minutos (momento 3) do término da perfusão; Grupo 2: perfusão esofágica com $\mathrm{HCl}$ 0,1 $\mathrm{N}$ e estudos eletromanométricos realizados 15 minutos após o término da perfusão (momento 2); Grupo 3: perfusão esofágica com $\mathrm{HCl}$ 0,1 N e estudos eletromanométricos realizados 30 minutos após o término da perfusão (momento 3). Resultados/Conclusão - Os resultados observados permitiram concluir que a acidificação do esôfago não provocou alterações significativas sobre a amplitude de pressão e comprimento do esfincter superior do esôfago.

DESCRITORES - Manometria. Junção esofagogástrica. Ácido clorídrico. Cães.

\footnotetext{
Professor Assistente do Departamento de Oftalmologia, Otorrinolaringologia e Cirurgia de Cabeça e Pescoço da Faculdade de Medicina de Botucatu, Universidade Estadual Paulista - UNESP, Botucatu, SP.

** Professor Titular do Departamento de Cirurgia e Ortopedia da Faculdade de Medicina de Botucatu.

*** Professor Adjunto do Departamento de Oftalmologia, Otorrinolaringologia e Cirurgia de Cabeça e Pescoço da Faculdade de Medicina de Botucatu.

Endereço para correspondência: Dr. José Vicente Tagliarini - Departamento de Oftalmologia, Otorrinolaringologia e Cirurgia de Cabeça e Pescoço - Faculdade de Medicina de Botucatu - UNESP - Distrito de Rubião Júnior s/n - 18618-000 - Botucatu, SP.
} 


\section{INTRODUÇÃO}

$\mathrm{O}$ esfíncter superior do esôfago (ESE) corresponde à zona de pressão intraluminar elevada que existe entre a faringe e o esôfago. Possui duas importantes funções:

a) constitui barreira ao refluxo do conteúdo gastroesofágico para as vias aerodigestivas superiores, protegendo assim a via respiratória de aspiração;

b) impede a entrada de ar no esôfago durante a inspiração ${ }^{(16)}$.

Estudos experimentais e clínicos têm investigado a ação do refluxo gastroesofágico sobre o ESE; entretanto, os resultados de tais pesquisas têm sido discordantes.

Enquanto alguns autores ${ }^{(8,9,20,27)}$ demonstraram contrações reflexas do ESE, com aumento da pressão durante episódios de refluxo gastroesofágico, outros discordam destes resultados ${ }^{(1,21,24)}$.

O objetivo do presente estudo foi estudar o efeito da acidificação esofágica sobre o ESE, utilizando o cão como animal de experimentação.

\section{MATERIAL E MÉTODO}

Foram utilizados 30 cães sem raça definida, de ambos os sexos, com pesos variando entre 10 e $15 \mathrm{~kg}$, submetidos a exame clínico prévio, tendo sido tratadas as afecções encontradas, bem como administrado vermífugo para erradicação de eventuais parasitoses intestinais.

Os animais foram estudados em duas etapas: na primeira foram realizados estudos eletromanométricos do ESE; a seguir, os cães foram submetidos a perfusão esofágica com água destilada ou $\mathrm{HCl} 0,1 \mathrm{~N}$, após o que, novos estudos motores do ESE foram efetuados. Os cães foram divididos em três grupos:

Grupo 1 (G1): perfusão esofágica com água destilada (grupo controle) e estudos eletromanométricos realizados antes e 15 e 30 minutos após o término da mesma.

Grupo 2 (G2): perfusão esofágica com ácido clorídrico $0,1 \mathrm{~N}$ e estudos eletromanométricos realizados antes e 15 minutos após o seu término.

Grupo 3 (G3): neste grupo, os animais foram submetidos a infusão esofágica com $\mathrm{HCl} 0,1 \mathrm{~N}$, sendo os estudos eletromanométricos realizados antes e 30 minutos após o término da perfusão.

Todos os procedimentos (eletromanometria e perfusão esofágica) foram realizados sob anestesia geral, usando-se pentobarbital sódico (Hipnol-Cristália), por via venosa, na dose de $30 \mathrm{mg} / \mathrm{kg}$ de peso do animal.

Para a eletromanometria foi utilizada sonda de três vias, confeccionada pela união de três cateteres de polietileno com 1,5 mm de diâmetro externo. Dos três cateteres, dois foram utilizados para a eletromanometria, sendo que os orifícios estavam situados no mesmo nível em orientação de $180^{\circ}$. O terceiro cateter tem por função a perfusão de água destilada ou de $\mathrm{HCl}$ na luz esofágica. O orifício utilizado para infusão esofágica dista $5 \mathrm{~cm}$ dos outros dois usados para as medidas pressóricas.
Para o registro das pressões, foram utilizados dois transdutores de pressão Elema Schonander, tipo capacitivo. O monitor de pressão digital permitiu a leitura de pressão nos dois canais e os registros gráficos foram realizados em fisiógrafo de dois canais da marca ECB (Equipamentos Científicos do Brasil). Para a infusão contínua dos cateteres, foi utilizado sistema de infusão contínua de baixa complacência do tipo pneumohidráulico com fluxo de $2,5 \mathrm{~mL}$ por minuto.

A bomba de infusão (modelo 680, marca Santronic) foi utilizada para perfusão esofágica. Esta foi realizada utilizando-se de um fluxo de 1,6 mL/min, evitando-se, assim, o estímulo volumétrico e eventual distensão do esôfago.

A eletromanometria foi realizada em três momentos:

M1: antes da perfusão esofágica

M2: 15 minutos após a perfusão

M3: 30 minutos após a perfusão

A eletromanometria foi realizada segundo a técnica de puxada intermitente da sonda, segundo padronização em nosso laboratório ${ }^{(3}$, $4,11,12,13,14,22)$. Nesta, a sonda foi introduzida até o terço inferior do esôfago e, a seguir, foi tracionada de um em um centímetro até atingir o ESE e neste, de meio em meio centímetro, até atingir a faringe. Após cada tração foi aguardada a estabilização do registro ${ }^{(26)}$

A eletromanometria permitiu o estudo da amplitude da pressão no ESE ( $\mathrm{mm} \mathrm{Hg}$ ) e do comprimento do ESE (cm).

Em cada momento de estudo (M1, M2 e M3), foram realizadas três medidas, efetuadas em dois canais, resultando 6 valores de cada atributo.

A perfusão esofágica foi realizada $5 \mathrm{~cm}$ abaixo do limite inferior do ESE, localizado através da eletromanometria. A bomba de infusão permitiu a perfusão de $20 \mathrm{~mL}$ de $\mathrm{HCl}$ ou água destilada durante 12 minutos (fluxo de 1,6 $\mathrm{mL}$ por minuto).

Em cada um dos momentos do estudo (M1, M2 e M3) foram calculados a média (x), o desvio padrão (s) e o coeficiente de variação $(\mathrm{CV})$ referentes aos dois atributos estudados.

A análise dos dados foi efetuada utilizando-se o método estatístico denominado análise de perfil ${ }^{(18)}$, que leva em consideração a estrutura de dependência entre valores medidos no mesmo animal, em diferentes momentos. A análise de perfil foi realizada com os testes de interação entre grupos e momentos ${ }^{(5)}$. Para o cálculo da estatística $F$ e seu respectivo nível de significância $P$, considerou-se significativo quando $P<0,05$.

\section{RESULTADOS}

Os valores médios, desvios padrão e coeficientes de variação referentes ao atributo amplitude da pressão no ESE (mm $\mathrm{Hg}$ ), estudados nos grupos 1, 2 e 3 estão expostos na Tabela 1 (valores médios na Figura 1)

A Tabela 2 mostra os valores médios do comprimento do ESE $(\mathrm{cm})$, desvios padrão e os coeficientes de variação referentes aos resultados observados nos grupos 1, 2 e 3 (valores médios na Figura 2). 
Tagliarini JV, Henry MACA, Bretan O. Eletromanometria do esfíncter superior do esôfago antes e após perfusão esofágica com ácido clorídrico $0,1 \mathrm{~N}$. Estudo experimental no cão

TABELA 1 - Amplitude da pressão no ESE (mm Hg). Médias, desvios padrão (DP) e coeficientes de variação (CV), observados nos três grupos experimentais e nos três momentos de estudo

\begin{tabular}{cccccccc}
\hline & \multicolumn{2}{c}{ Antes } & \multicolumn{2}{c}{$\mathbf{1 5}$ minutos } & \multicolumn{2}{c}{ 30 minutos } \\
\hline Grupo & Média \pm DP & CV (\%) & Média \pm DP & CV (\%) & Média \pm DP & CV (\%) \\
1 & $12,0 \pm 5,6$ & 46,6 & $11,5 \pm 6,6$ & 57,1 & $16,5 \pm 11,6$ & 69,9 \\
2 & $7,5 \pm 3,0$ & 40,4 & $8,9 \pm 6,6$ & 62,6 & - & - \\
3 & $11,8 \pm 6,8$ & 58,0 & - & - & $14,5 \pm 9,6$ & 66,0 \\
\hline
\end{tabular}

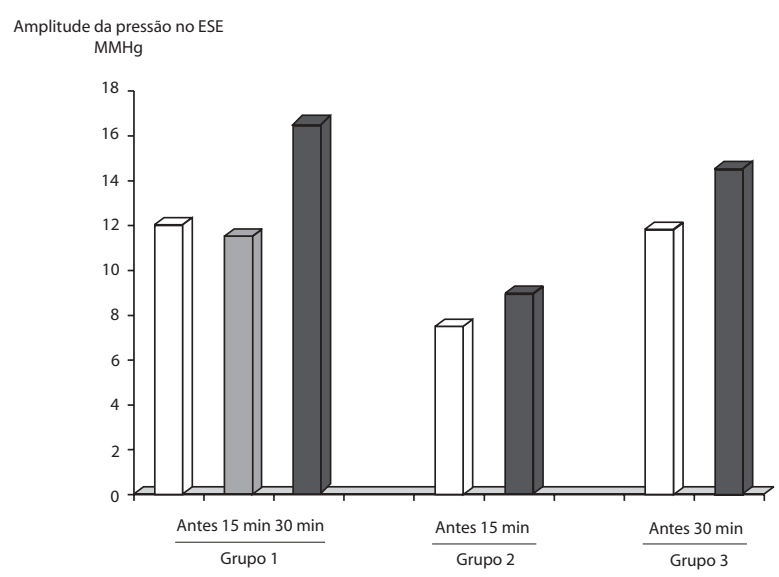

FIGURA 1 - Amplitude da pressão no ESE: valores médios observados nos três grupos experimentais

TABELA 2 - Comprimento do ESE (cm). Médias, desvios padrão (DP) e coeficientes de variação (CV), observados nos três grupos experimentais e nos três momentos de estudo

\begin{tabular}{ccccccc}
\hline & \multicolumn{2}{c}{ Antes } & \multicolumn{2}{c}{$\mathbf{1 5}$ minutos } & \multicolumn{2}{c}{ 30 minutos } \\
\hline Grupo & Média \pm DP & CV (\%) & Média \pm DP & CV (\%) & Média \pm DP & CV (\%) \\
1 & $1,9 \pm 0,3$ & 16,0 & $1,6 \pm 0,3$ & 19,6 & $1,8 \pm 0,4$ & 23,5 \\
2 & $1,5 \pm 0,5$ & 31,5 & $1,6 \pm 0,2$ & 29,7 & - & - \\
3 & $2,0 \pm 0,5$ & 25,4 & - & - & $2,0 \pm 0,6$ & 29,6 \\
\hline
\end{tabular}

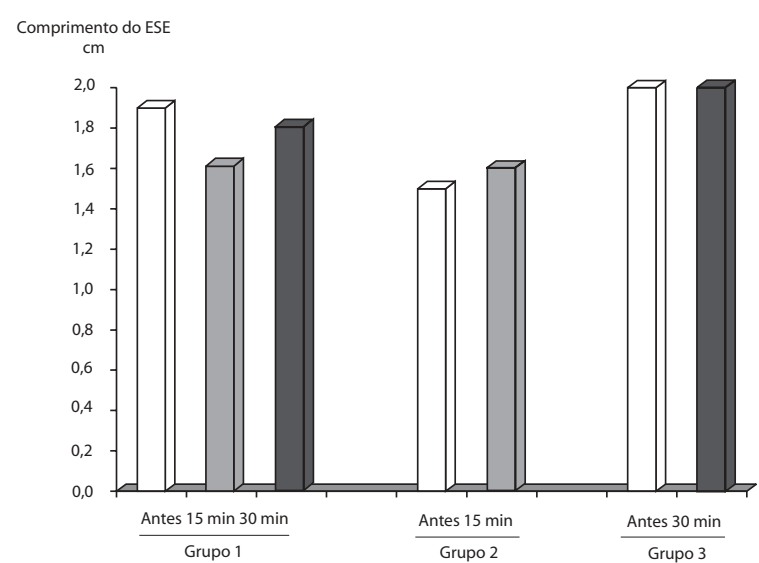

FIGURA 2 - Comprimento do ESE: valores médios observados nos três grupos experimentais. 


\section{Análise dos Resultados}

Os valores médios de pressão do ESE, bem como seu comprimento, observados após 15 e 30 minutos da perfusão de água destilada ou $\mathrm{HCl} 0,1 \mathrm{~N}$ não diferiram dos valores medidos antes da perfusão.

\section{DISCUSSÃO}

A perfusão do esôfago com ácido clorídrico tem sido estudada em inúmeras pesquisas experimentais. Na presente, o animal escolhido foi o cão, por se tratar de animal de fácil obtenção em nosso biotério ${ }^{(23,}$ ${ }^{25)}$. Além disso, o cão é animal dócil, sendo possível sua utilização em estudos motores neste laboratório ${ }^{(3,11,22,23,25)}$.

A perfusão esofágica com $\mathrm{HCl}$ ou água destilada foi realizada usando-se bomba de perfusão que permitiu fluxo de $1,6 \mathrm{~mL} / \mathrm{min}$, semelhante ao relatado em várias pesquisas experimentais ${ }^{(7,10)}$. Nos trabalhos clínicos o fluxo utilizado é mais elevado, tendo em vista as dimensões do esôfago humano ${ }^{(1,20)}$. A perfusão foi realizada $5 \mathrm{~cm}$ abaixo do ESE, levando em conta os relatos de alguns autores que obtiveram respostas pressóricas mais evidentes quando a perfusão foi realizada no terço proximal do esôfago torácico ${ }^{(8,9)}$.

Para o estudo motor do esôfago, foi utilizado neste trabalho o sistema de perfusão contínua do tipo pneumohidráulico de baixa complacência, cuja eficiência tem sido demonstrada por vários autores ${ }^{(2,19)}$.

A eletromanometria foi realizada através da técnica de puxada intermitente da sonda, usada em inúmeros trabalhos experimentais ${ }^{(14,}$ 23) e clínicos ${ }^{(6,15)}$.

A amplitude da pressão o ESE medida em condições basais apresentou, na presente pesquisa, os valores $12,0 \pm 5,6 \mathrm{~mm} \mathrm{Hg}, 7,5 \pm$ $3,0 \mathrm{~mm} \mathrm{Hg}$ e $11,8 \pm 6,8 \mathrm{~mm} \mathrm{Hg}$ para os grupos 1,2 e 3, respectivamente. Estes valores são semelhantes aos relatados por FREIMAN et al. ${ }^{(8)}$, porém inferiores aos de LYNCH et al. ${ }^{(17)}$ e de BRETAN $^{(3)}$. A variabilidade observada no presente experimento para os valores da amplitude da pressão no ESE esteve entre $40 \%$ e $57 \%$, mais elevados que os relatados por BRETAN ${ }^{(3)}$.

A perfusão esofágica com água destilada (Grupo 1) não acarretou qualquer alteração na amplitude da pressão no esfíncter superior do esôfago, quer nas medidas realizadas 15 minutos após a infusão (momento 2), ou naquelas observadas após 30 minutos (momento 3 ). Este resultado já era esperado por se tratar do grupo controle.
Nos grupos 2 e 3 foi analisado o efeito da perfusão de $\mathrm{HCl} 0,1 \mathrm{~N}$ sobre o ESE, não tendo sido observada qualquer alteração do mesmo, em medidas realizadas 15 e 30 minutos após a perfusão (momentos 2 e 3, respectivamente). Estes resultados diferem dos publicados por FREIMAN et al. ${ }^{(8)}$, pois estes autores demonstraram que a acidificação do esôfago de três cães acarretou aumento da pressão do ESE.

Pesquisas realizadas em voluntários ${ }^{(9,27)} \mathrm{e}$ em crianças portadoras de refluxo gastroesofágico ${ }^{(20)}$, também demonstraram aumento da pressão no esfíncter superior do esôfago, resultado discordante do observado na presente investigação.

ANDREOLLO et al. ${ }^{(1)}$, após realizarem acidificação do esôfago em sete indivíduos normais, também observaram aumento da pressão no ESE. Resultado idêntico foi obtido também pela perfusão esofágica com soro fisiológico, fato que levou os autores a concluírem que a resposta do esfíncter é devida à distensão provocada pelo fluído na parede do esôfago e não à presença do ácido.

Na presente investigação foi utilizado na perfusão esofágica, fluxo de apenas 1,6 mL/minuto, a fim de se evitar distensão da parede do esôfago. É provável que a ausência de resposta do esfíncter superior do esôfago seja decorrente deste fato.

Pesquisas semelhantes foram realizadas em indivíduos normais e portadores da doença do refluxo gastroesofágico ${ }^{(21,24)}$. Estes autores não observaram alteração da amplitude da pressão do esfíncter superior do esôfago, resultado idêntico ao desta pesquisa experimental.

O comprimento do ESE foi medido pela técnica de puxada intermitente da sonda e, em condições basais, foram observados os seguintes resultados: $1,9 \pm 0,3 \mathrm{~cm}, 1,5 \pm 0,5 \mathrm{~cm}$ e $2,0 \pm 0,5 \mathrm{~cm}$ para os grupos 1, 2 e 3, respectivamente. Os resultados são comparáveis aos publicados por LYNCH et al. ${ }^{(17)}$ e BRETAN ${ }^{(3)}$, em trabalhos realizados no cão. A variabilidade deste parâmetro em condições basais apresentou valores entre $16 \%$ e $31,5 \%$, semelhantes aos de BRETAN ${ }^{(3)}$ e UVO ${ }^{(23)}$, em trabalhos realizados no cão e no coelho, respectivamente.

A perfusão esofágica com água destilada (grupo 1) e com $\mathrm{Hcl}$ $0,1 \mathrm{~N}$ (grupos 2 e 3 ) não acarretou qualquer alteração no comprimento do ESE. Não foram encontrados na literatura trabalhos experimentais ou clínicos com o objetivo de estudar este atributo após acidificação esofágica, fato que dificulta a comparação de nossos resultados.

Os resultados do presente estudo nos levam a concluir que a acidificação esofágica não altera a pressão e o comprimento do ESE nos momentos da investigação. 
Tagliarini JV, Henry MACA, Bretan O. Eletromanometria do esfíncter superior do esôfago antes e após perfusão esofágica com ácido clorídrico $0,1 \mathrm{~N}$. Estudo experimental no cão

Tagliarini JV, Henry MACA, Bretan O. Electromanometry study of the upper esophageal sphincter before and after esophageal infusion with HCl 0,1N. Experimental study in dog. Arq Gastroenterol 2001;38(3):189-193.

ABSTRACT - Background - The responses of the upper esophageal sphincter to gastroesophageal reflux is controversial. Objective - Study the effect of upper esophageal sphincter to the esophageal acid perfusion. Methods - Thirty adult dogs of both sexes were studied, being submitted to esophageal electromanometry. The pull through technique and continuous infusion of the catheters with distilled water were employed. These exams allowed us to measure the pressure width $(\mathrm{mm} \mathrm{Hg})$ and the length $(\mathrm{cm})$ of the upper esophageal sphincter in basal conditions (moment 1). After this first phase the animals were submitted to esophagic infusion, being then divided in three groups, according to the solution used in the infusion and the moment of the study, as follows: Group 1: esophagic infusion with distilled water and electromanometric studies accomplished 15 minutes (moment 2) and 30 minutes (moment 3) of the end of the infusion. Group 2: esophagic infusion with $\mathrm{HCl}$ 0,1 $\mathrm{N}$ and electromanometric studies accomplished 15 minutes after the end of the infusion (moment 2). Group 3: esophagic infusion with $\mathrm{HCl} \mathrm{0,1} \mathrm{N}$ and electromanometric studies accomplished 30 minutes after the end of the infusion (moment 3). Results/Conclusions - This research was performed to evaluate the esophagic acidification influence on the upper esophageal sphincter. The observed results allowed us to conclude that the acidification of the esophagus did not cause any alteration on the pressure width and on the length of the upper esophageal sphincter.

HEADINGS - Manometry. Esophagogastric junction. Hydrochloric acid. Dogs.

\section{REFERÊNCIAS BIBLIOGRÁFICAS}

1. Andreollo NA, Thompson DG, Kendall GPN, McIntyre AS, Earlam RJ. Motor responses of the upper esophageal sphincter and body to intraluminal acid. Braz J Med Biol Res 1989;22:51-60.

2. Arndorfer RC, Stef JJ, Dodds WJ, Linehan JH, Hogan WJ. Improved infusion system for intraluminal esophageal manometry. Gastroenterology 1977;73:237 .

3. Bretan O. Eletromanometria do esfíncter superior do esôfago e suas relações com o esfíncter inferior: estudo experimental no cão [tese de doutorado em Cirurgia Experimental]. Botucatu: Faculdade de Medicina de Botucatu da Universidade Estadual Paulista; 1986. 197p.

4. Bretan O, Henry MAC, Cury PR. Techniques in electromanometry of upper esophageal sphincter. Arch Otolaryngol Head Neck Surg 1990;116:914-6.

5. Curi PR. Metodologia e análise da pesquisa em ciências biológicas. Botucatu: Editora Tipomic; 1997. 263p.

6. Dantas RO, Aguiar LN, Ramos FS, Mamede RCM, Silva AK, Gonçalves AS, Mello-Filho FV. Evaluation of esophageal motility in laryngectomized patients. Arq Gastroenterol 1999;36:112-6.

7. Eastwood GL, Castell DO, Higgs RH. Experimental esophagitis in cats impairs lower esophageal sphincter pressure. Gastroenterology 1975;69:146-50.

8. Freiman JM, El-Sarkamy TY, Diamant NE. Effect of bilateral vagosympathetic nerve blockage on response of the dog upper esophageal sphincter to intraesophageal distension and acid. Gastroenterology 1981;81:78-84.

9. Gerhardt DC, Shuck TJ, Bordeaux RA, Winship DH. Human upper esophageal sphincter. Response to volume, osmotic and acid stimuli. Gastroenterology 1978;75:268-74.

10. Goldberg HI, Dodds WJ, Gee S, Montgomery C, Zboralske F. Role of acid and pepsin in acute experimental esophagitis. Gastroenterology 1969;56:223-30.

11. Henry MACA. Eletromanometria e pHmetria da junção gastroesofágica: estudo experimental no cão [tese de doutorado em Cirurgia Experimental]. Botucatu: Faculdade de Medicina de Botucatu da Universidade Estadual Paulista; 1979. $178 \mathrm{p}$.

12. Henry MACA, Vercesi LAP, Lucchiari PH. L'action du thiopental sur le oesophagien inferieur. Cah Anesthesiol 1984;32:37-8.

13. Henry MACA, Leite CVS, Mendes EF. Influência da vagotomia gástrica proximal sobre a zona de alta pressão esôfago-gástrica no cão. Arq Gastroenterol $1988 ; 25: 188-92$.
14. Henry MACA, Athanasio EYU. Efeito da metoclopramida, da ranitidina e do droperidol sobre o esfíncter inferior do esôfago. Estudo experimental no gambá (Didelphis allbiventris). Arq Gastroenterol 1994;31:103-7.

15. Henry MACA, Prado RG. Emprego da toxina botulínica no tratamento do espasmo difuso esofagiano. Relato de caso. Arq Gastroenterol 1998;35:274-7.

16. Kilman WJ, Goyal RK. Disorders of pharyngeal and upper esophageal sphincter motor function. Arch Intern Med 1976;136:592-601.

17. Lynch VF, Schlegel JF, Ellis FH Jr. Effects of denervation and muscle ablation procedures on the canine pharyngoesophageal junction. Thorax 1973;28:228-34

18. Morrison DF. Multivariate statistical methods. New York: Mc Graw Hill; 1967. $338 \mathrm{p}$.

19. Pope II CE. A dynamic test of sphincter strength: its application to the lower esophageal sphincter. Gastroenterology 1967;52:779-86.

20. Sondheimer JM. Upper esophageal sphincter and pharyngoesophageal motor function in infants with and without gastroesophageal reflux. Gastroenterology 1983;85:301-5.

21. Stanciu C, Bennet JR. Upper esophageal sphincter yield pressure in normal subjects and in patients with gastroesophageal reflux. Thorax 1974;29:459-62.

22. Uvo SAB. Efeito da laringectomia horizontal sobre a pressão e comprimento dos esfíncteres superior e inferior do esôfago: estudo experimental no coelho [tese de mestrado em Cirurgia Experimental]. Botucatu: Faculdade de Medicina de Botucatu da Universidade Estadual Paulista; 1991. 120p.

23. Uvo SAB, Henry MACA, Bretan O. Functional evaluation of the iliocolic sphincter transplanted to the neck using microsurgery technique: a manometric study in dogs. Dis Esophagus 1999;12:10-3.

24. Vakil NB, Kahrilas PJ, Dodds WJ, Vanagunas A. Absence of an upper esophageal sphincter response to acid reflux. Am J Gastroenterol 1989;84:606-10.

25. Vercesi LAP. Estudo eletromanométrico com infusão constante da zona alta pressão esofagogástrica no cão [tese de doutorado em Cirurgia Experimental]. Botucatu: Faculdade de Ciências Médicas e Biológicas da Universidade Estadual Paulista; 1972. 85p.

26. Vercesi LAP, Henry MAC, Hossne WS. Estabilização do registro, novo fenômeno observado nos registros eletromanométricos da zona de alta pressão esofagogástrica (ZAP) no cão. Arq Gastroenterol 1982;19:73-6.

27. Wallin L, Boesby S, Madsen T. The effect of the $\mathrm{HCl}$ infusion in the pharyngoesophageal sphincter pressure in normal subjects. Scand J Gastroenterol 1978;13:821-6.

Recebido em 3/8/2000 Aprovado em 10/11/2000 